\title{
COMPARISON OF SIGNIFICANCE OF LIPID PROFILE WITH LIVER FUNCTION TESTS IN DENGUE FEVER
}

\author{
Santhosh ${ }^{1}$, Indumathi Shankaraiah², Harish Kiran ${ }^{3}$
}

${ }_{1}^{1}$ Assistant Professor, Department of Medicine, MVJ Medical College, Hoskote, Karnataka, India.

${ }^{2}$ Assistant Professor, Department of Medicine, Subbaiah Medical College, Shimoga, Karnataka, India.

${ }^{3}$ Assistant Professor, Department of Radiology, Subbaiah Medical College, Shimoga, Karnataka, India.

\section{ABSTRACT}

\section{BACKGROUND}

Dengue is the most prevalent mosquito borne viral infection with rapidly changing epidemiology over the last few years. Lipoproteins play a pathophysiological role in the hosts immune response to severe infection. The need to know the lipoprotein status in dengue fever, find its correlation with severity, liver function tests, and clinical outcome are the objectives of this study.

\section{METHODS}

This prospective observational study was taken up involving 50 adults diagnosed with dengue fever between December 2013 to December 2014 in Mysore Medical College. Patients were classified based on severity of dengue fever (DF), dengue haemorrhagic fever (DHF), dengue shock syndrome (DSS). Fasting lipid profile, liver function test (LFT), complete blood count were estimated on day 1 and day 5 of admission.

\section{RESULTS}

The study population comprised of 50 dengue sero positive cases. The total cholesterol was greatly reduced and LFT alterations were seen highest in DSS group. During admission, serum cholesterol and liver enzymes showed statistically significant difference in their mean concentrations in the three groups of dengue infection. The spearman correlation analysis shows that AST and total cholesterol had negative correlation $(r=-0.782 ; \mathrm{p}<0.01)$, whereas ALT and cholesterol also showed similar correlation $(\mathrm{r}=-0.738$; $\mathrm{p}<0.01)$.

\section{CONCLUSIONS}

Total cholesterol, HDL and LDL of dengue fever cases were significantly reduced at admission in comparison to the second estimation on day 5. Mean cholesterol, triglyceride and LDL levels showed statistically significant $(p$ value $<0.05)$ changes with severity of dengue. LFT was correlated with the severity of dengue fever. These abnormal values may serve as indicators for recognizing patients at increased risk for DHF and DSS, thus serving as prognostic markers of the dengue infected individuals.

HOW TO CITE THIS ARTICLE: Santhosh, Shankaraiah I, Kiran H. Comparison of significance of lipid profile with liver function tests in dengue fever. J. Evolution Med. Dent. Sci. 2019;8(14):1169-1173, DOI: 10.14260/jemds/2019/259

\section{BACKGROUND}

Dengue is an acute viral infection hyper-endemic in tropics where significant population is at risk.[1] The World Health Organization (WHO) estimated that 50 million dengue infections occur annually, making it as the most rapidly spreading arbo viral infection. [2] Dengue is caused by RNA virus of the genus Flavi virus. There are four distinct serotypes called dengue virus (DENV) type 1 to 4 and it is transmitted to humans through the bites of infected Aedes mosquitoes, mainly by female Aedes Aegypti as well as Aedes Albopictus.[3]

The nonspecific febrile illness with mild flu-like syndrome is known as dengue fever (DF). Infection with any of the DENV serotypes can result in a wide spectrum of symptoms, characterized by coagulopathy, increased vascular fragility and permeability, leading to manifestation of capillary leak and haemorrhage, known as dengue haemorrhagic fever (DHF). In severe cases, the increased vascular permeability

'Financial or Other Competing Interest': None.

Submission 14-02-2019, Peer Review 21-03-2019,

Acceptance 28-03-2019, Published 08-04-2019.

Corresponding Author:

Indumathi Shankaraiah,

\#S3, A-Block, Teaching Faculty Quarters,

Subbaiah Institute of Medical Sciences,

NH-13, Purlae, H. H. Road, Shimoga-577222, Karnataka, India.

E-mail: drindu126@gmail.com

DOI: $10.14260 /$ jemds $/ 2019 / 259$

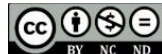

leads to circulatory compromise and the patient may develop potentially life-threatening dengue shock syndrome (DSS).[4] The more complicated forms of dengue also present with fever and initial symptoms are indistinguishable from the benign DF thus leading to delayed diagnosis of DHF and DSS. Hence, it is important to explore prognostic markers of severe disease during acute phase of the infection. The morbidity and the mortality of DHF can be reduced by early diagnosis, hospitalization and symptomatic care.

Liver is one of the target organs of dengue virus, and its involvement can be diagnosed by hepatomegaly and an increase in hepatic enzymes, in serum after infection. Hepatic dysfunction is well recognized feature of dengue infections, often demonstrated by mild-to moderate increases in transaminase levels although jaundice and acute liver failure (ALF) are uncommon.[5] Lipid profile changes accompany dengue infection, some of which may indicate severity and guide therapy. Cytokines induced by severe infections bring about changes in the lipoprotein levels which plays a pathophysiological role in the host's immune response. In patients with bacterial infection, lipoproteins, including very low-density lipoprotein (VLDL), bind endotoxin and neutralize the toxic effects of endotoxin. Interactions between microorganisms and lipoproteins also occur in viral infections. Certain viruses use low-density lipoprotein (LDL) receptors to enter the cell, which implies that LDL may compete with viruses for these cellular receptors. Therefore, high LDL levels may be beneficial because they decrease virus 
uptake by cells. Lipoproteins also bind viruses and neutralize their toxic effects.[6]

Changes in serum lipid profile and lipid metabolism during dengue infection and its underlying biological mechanisms remain unclear. Therefore, this study was undertaken with an aim at evaluating the prognostic significance of lipid profile amongst dengue cases by comparing it with liver function tests of the patients.

\section{Aim of The Study}

To predict the severity in Dengue fever by correlating Biochemical alteration in Lipid Profile and Liver Function test with the clinical outcome.

\section{Study Objectives}

1. To determine Lipid profile alteration in Dengue cases.

2. To determine Liver Function Test alteration in Dengue cases.

3. To compare the Biochemical parameters in Dengue fever, dengue haemorrhagic fever and Dengue shock syndrome.

\section{METHODS}

\section{Study Design}

This prospective observational study was taken up involving 50 adults diagnosed with dengue fever and admitted to $\mathrm{K} \mathrm{R}$ Hospital attached to Mysore Medical College and Research Institute between December 2013 to December 2014. Patients were classified according to WHO criteria based on the severity of presentation and were grouped as:

\section{Group 1}

Dengue fever (DF)

Group 2

Dengue Haemorrhagic Fever (DHF)

Group 3

Dengue Shock Syndrome (DSS)

All patients were included into the study after obtaining written informed patient consent Ethical clearance was obtained from the Institutional Ethics Committee.

Sample size has been taken based on the convenience of the study

\section{Inclusion Criteria}

Adults diagnosed as having dengue fever on basis of IgM ELISA test for dengue fever.

\section{Exclusion Criteria}

1. Patients with diabetes mellitus and hypertension.

2. Patients on lipid lowering drugs, corticosteroids, oral contraceptives, antitubercular therapy.

3. Patients with history of ischemic heart disease, congestive cardiac failure, chronic renal failure, chronic liver disease.

4. Pregnant women.

5. Alcoholic liver disease.

6. Blood coagulation disorders.

7. Nephrotic syndrome.

Detailed history was taken and meticulous clinical examination was carried out according to the pre-texted protocol. Information from all patients regarding any co- morbid conditions, medications being taken and past dengue infection was also collected. Fasting serum lipid profile and liver functions was tested on Day of admission, Day 1. Second estimation of all the same parameters was done on Day 5 of hospitalization. Serum triglycerides, cholesterol, HDL were estimated. LDL and VLDL were calculated using Friedwald's formula.

\section{Methodology}

The data was presented as mean and standard deviation. Statistical analysis was done using SPSS 16.0 version, IBM.

\section{RESULTS}

The study population comprised of 50 Dengue seropositive cases amongst whom 31 were females and 19 males. The average age of the cases was $55 \pm 15$ years. The study subjects were divided into 3 groups:

\section{Group I}

24 cases of dengue fever.

\section{Group II}

17 cases of dengue haemorrhagic fever.

\section{Group III}

9 cases of dengue shock syndrome.

\begin{tabular}{|c|c|c|}
\hline & At Admission & Recovery Phase \\
\hline Haematocrit & 44.69 & 45.45 \\
\hline Haemoglobin $(\mathrm{g} / \mathrm{dL})$ & 12.73 & 14.24 \\
\hline Red Blood Cell Count & 4.71 & 5.01 \\
\hline White Blood Cell Count & 5.56 & 7.41 \\
\hline Platelet Count & 58.8 & 195.73 \\
\hline
\end{tabular}

\begin{tabular}{|c|c|c|c|}
\hline Parameters & At Admission & on Day 5 & p Value \\
\hline Total Protein & $6.45 \pm 0.58$ & $6.79 \pm 0.51$ & 0.000 \\
\hline Albumin & $3.69 \pm 0.84$ & $3.77 \pm 0.4$ & 0.001 \\
\hline Total Bilirubin & $2.16 \pm 1.3$ & $3.77 \pm 0.4$ & 0.0000 \\
\hline Direct Bilirubin & $1 \pm 0.7$ & $0.53 \pm 0.3$ & 0.0000 \\
\hline AST & $115 \pm 60$ & $37 \pm 20$ & 0.000 \\
\hline ALT & $80 \pm 49$ & $31 \pm 19$ & 0.001 \\
\hline ALP & $119 \pm 44$ & $69 \pm 21$ & 0.000 \\
\hline Creatinine & $0.94 \pm 0.12$ & $0.87 \pm 0.09$ & 0.000 \\
\hline $\begin{array}{c}\text { Blood Urea } \\
\text { Nitrogen }\end{array}$ & $36.3 \pm 12.4$ & $25.9 \pm 9.12$ & 0.000 \\
\hline $\begin{array}{c}\text { Total Cholesterol } \\
\text { Triglycerides }\end{array}$ & $189 \pm 17.16$ & $194.88 \pm$ & 19.75 \\
\hline HDL & $40 \pm 10$ & $39 \pm 9$ & 0.041 \\
\hline LDL & $119 \pm 12.94$ & $121 \pm 17.7$ & 0.224 \\
\hline VLDL & $26 \pm 9$ & $25 \pm 9$ & 0.672 \\
\hline
\end{tabular}

Table 2. Biochemical Investigations of Dengue Fever Patients on Day 1 and Day 5 Wilcoxon Summed Rank Test $p$ value $<0.05$ is statistically significant

\begin{tabular}{|c|c|c|c|}
\hline Parameters & At Admission & On Day 5 & p Value \\
\hline Total Protein & $6.27 \pm 0.72$ & $6.6 \pm 0.64$ & 0.001 \\
\hline Albumin & $3.59 \pm 0.39$ & $3.88 \pm 0.37$ & 0.000 \\
\hline Total Bilirubin & $4.48 \pm 2.49$ & $1.56 \pm 0.89$ & 0.000 \\
\hline Direct Bilirubin & $2.33 \pm 1.65$ & $1.08 \pm 1.25$ & 0.001 \\
\hline AST & $327 \pm 203$ & $115 \pm 125$ & 0.000 \\
\hline
\end{tabular}


LCAT. During the acute stage of infections, serum triglyceride concentration increases, whereas HDL cholesterol and LDL cholesterol concentrations decrease. Interestingly, whereas serum LDL cholesterol concentration declines because of infection, the proportion of small, dense LDL particles considered to be more atherogenic than larger LDL increases.[12] Kruskal Wallis test done for lipid profile at the time of admission showed statistically significant alterations between total cholesterol, HDL and LDL between the three groups. The triglyceride was not statistically significant between the 3 groups.

The liver is one of the target organs for dengue and clinical manifestations of hepatic dysfunction can occur during the course of this disease. Severity of hepatic dysfunction is positively correlated to increased severity of dengue in patients. The dengue virus is able to replicate in both hepatocytes and Kupffer cells. Receptor-mediated endocytosis and fusion aids in virus replication in Kupffer cells. Kupffer cell activation releases inflammatory cytokines. Our patients had markedly elevated serum transaminases at values above 10 times the upper limit of normal and the AST levels were higher than ALT as found in the few case reports of dengue associated ALF.[13] Dengue infection can cause acute damage to these non-hepatic tissue types that express AST, raised aminotransferase levels may not be entirely due to severe liver involvement. It is therefore possible that the patients with high AST levels were also more likely to be classified as severe dengue under the 2009 criteria due to the common pathways to non-hepatic tissue damage, even though there is no association with poorer outcome.[14] This additional non-hepatic source of AST like myocytes could explain the pattern observed.

Dengue fever is usually associated with mild to moderate elevations of aminotransferase levels Liver damage was more frequent among women, patients with sequential infection and hemorrhagic cases. Use of hepatotoxic drugs have the potential to aggravate liver damage, therefore should be strictly monitored.[15]

A study by Felmlee DJ et al pointed that inhibition of the cholesterol synthetic pathway by inhibiting the rate-limiting step 3-hydroxy-methyl glutaryl CoA reductase completely disrupted viral replication.[16] Blockage of the cholesterol synthetic pathway affects not only cholesterol synthesis but also other processes, such as the synthesis of isoprenoids, lipid intermediates necessary for protein prenylation; dolichol phosphate, which is involved in protein glycosylation; and ubiquinone, which is involved in respiratory chain activity. Such multiple mechanisms have been basis for putting forth the theory that statins may help in controlling dengue infection by Gutriezz et al.[17]

This study is prospective in nature, where some of the previous studies were retrospective or cross sectional in nature. Study group included seronegative dengue cases whereas this study has been done on seropositive patients. The lipid profile and liver function tests of the study group were estimated. Therefore, utmost care was taken to exclude other common disorders which affected these biochemical parameters by detailed history taking before including patients into this study.

\section{Limitations}

This study is a hospital-based study with a small sample size, therefore the extrapolation of this result to the general population needs further studies with larger sample size.

\section{CONCLUSIONS}

1. Female preponderance was seen, which is $62 \%$. Male being only $38 \%$.

2. Total cholesterol, HDL, LDL and VLDL are reduced during admission of all Dengue cases.

3. Total cholesterol, HDL, LDL and VLDL decreased with increasing severity.

4. Triglyceride concentrations elevated with increased severity of infection.

5. Total cholesterol was severely reduced in Dengue shock syndrome compared to other groups (DF, DHF).

6. AST and total cholesterol showed negative correlation.

7. ALT and total cholesterol also showed negative correlation.

8. Transaminase value showed 5 fold higher than normal values in both DF and DHF.

Lipid profile changes accompany dengue infection, some of which may indicate severity and guide therapy. Consecutive estimation of the same parameters on day five of hospitalization showed statistically significant differences in total cholesterol levels across the three groups. The lipid profile of subjects showcased the tendency to approach normal levels during recovery phase. This reversible trend suggests dengue fever as a potential cause for lipid alteration. These abnormal values may serve as indicators for recognition of patients at increased risk for DHF and DSS, thus serving as prognostic markers of the dengue infected individuals.

\section{REFERENCES}

[1] Lee IK, Liu JW, Yang KD. Fatal dengue hemorrhagic fever in adults: emphasizing the evolutionary pre-fatal clinical and laboratory manifestations. PLoS Negl Trop Dis 2012;6(2):e1532.

[2] Tan SS, Bujang MA. The clinical features and outcomes of acute liver failure associated with dengue infection in adults: a case series. Braz J Infect Dis 2013;17(2):164-9.

[3] Parkash O, Almas A, Jafri SMW, et al. Severity of acute hepatitis and its outcome in patients with dengue fever in a tertiary care hospital Karachi, Pakistan (South Asia). BMC Gastroenterol 2010;10:43.

[4] Jyothi P, Metri BC. Correlation of serological markers and platelet count in the diagnosis of Dengue virus infection. Adv Biomed Res 2015;4:26.

[5] Trung DT, Thao le TT, Hien TT, et al. Liver involvement associated with dengue infection in adults in Vietnam. Am J Trop Med Hyg 2010;83(4):774-80.

[6] Chakravarti A, Kumaria R. Eco-epidemiological analysis of dengue infection during an outbreak of dengue fever, India. Virol J 2005;2:32. 
[7] Clyde K, Kyle JL, Harris E. Recent advances in deciphering viral and host determinants of dengue virus replication and pathogenesis. J Virol 2006;80(23):11418-31.

[8] Zhang F, Kramer CV. Corticosteroids for dengue infection. Cochrane Database Syst Rev 2014;(7):CD003488.

[9] Villar-Centeno LA, Díaz-Quijano FA, Martínez-Vega RA. Biochemical alterations as markers of dengue hemorrhagic fever. Am J Trop Med Hyg 2008;78(3):370-4.

[10] Bansal D, Bhatti HS, Sehgal R. Role of cholesterol in parasitic infections. Lipids Health Dis 2005;4:10.

[11] Belay E, Seifu D, Amogne W, et al. Lipid profile derangements among human immunodeficiency virus infected adults receiving first line anti-retroviral therapy in Tikur Anbesa specialized hospital, Addis Ababa, Ethiopia: comparative cross-sectional study. J AIDS \& Clin Res 2014;5(8):328.

[12] Seneviratne SL, Malavige GN, de Silva HJ. Pathogenesis of liver involvement during dengue viral infections. Trans R Soc Trop Med Hyg 2006;100(7):608-14.
[13] Souza LJ, Coelho JM, Silva EJ, et al. Acute hepatitis due to dengue virus in a chronic hepatitis patient. Braz J Infect Dis 2008;12(5):456-9.

[14] Lee LK, Gan VC, Lee VJ, et al. Clinical relevance and discriminatory value of elevated liver aminotransferase levels for dengue severity. PLoS Negl Trop Dis 2012;6(6):e1676.

[15] Souza LJ, Alves JG, Nogueira RM, et al. Aminotransferas changes and acute hepatitis in patients with dengue fever: analysis of 1, 585 cases. Braz J Infect Dis 2004;8(2):156-63.

[16] Felmlee DJ, Hafirassou ML, Lefevre M, et al. Hepatitis C virus, cholesterol and lipoproteins--impact for the viral life cycle and pathogenesis of liver disease. Viruses 2013;5(5):1292-324.

[17] Martínez-Gutierrez M, Castellanos JE, Gallego-Gómez JC. Statins reduce dengue virus production via decreased virion assembly. Intervirology 2011; 54(4):202-16. 